\title{
SPECTRAL BAND SELECTION FOR URBAN MATERIAL CLASSIFICATION USING HYPERSPECTRAL LIBRARIES
}

\author{
A. Le Bris ${ }^{\mathrm{a} *}$, N. Chehata ${ }^{\mathrm{b}, \mathrm{c}}$, X. Briottet ${ }^{\mathrm{d}}$, N. Paparoditis ${ }^{\mathrm{a}}$ \\ a Université Paris-Est, IGN/SR, MATIS, 73 avenue de Paris, 94160 Saint Mandé , France - (arnaud.le-bris, nicolas.paparoditis)@ign.fr \\ ${ }^{\mathrm{b}}$ IRD/UMR LISAH El Menzah 4, Tunis, Tunisia \\ ${ }^{c}$ Bordeaux INP, G\&E, EA 4592, F-33600, Pessac, France - nesrine.chehata@ensegid.fr \\ ${ }^{\mathrm{d}}$ ONERA, The French Aerospace Lab, 2 avenue Edouard Belin, BP 74025, 31055 Toulouse Cedex 4, France - xavier.briottet@ onera.fr
}

Commission VII, WG VII/3

KEY WORDS: Classification, Feature selection, Sensor design, Hyperspectral, Spectral bands, Multispectral, Urban materials, Spectral libraries, SWIR

\begin{abstract}
:
In urban areas, information concerning very high resolution land cover and especially material maps are necessary for several city modelling or monitoring applications. That is to say, knowledge concerning the roofing materials or the different kinds of ground areas is required. Airborne remote sensing techniques appear to be convenient for providing such information at a large scale. However, results obtained using most traditional processing methods based on usual red-green-blue-near infrared multispectral images remain limited for such applications. A possible way to improve classification results is to enhance the imagery spectral resolution using superspectral or hyperspectral sensors. In this study, it is intended to design a superspectral sensor dedicated to urban materials classification and this work particularly focused on the selection of the optimal spectral band subsets for such sensor. First, reflectance spectral signatures of urban materials were collected from 7 spectral libraires. Then, spectral optimization was performed using this data set. The band selection workflow included two steps, optimising first the number of spectral bands using an incremental method and then examining several possible optimised band subsets using a stochastic algorithm. The same wrapper relevance criterion relying on a confidence measure of Random Forests classifier was used at both steps. To cope with the limited number of available spectra for several classes, additional synthetic spectra were generated from the collection of reference spectra: intra-class variability was simulated by multiplying reference spectra by a random coefficient. At the end, selected band subsets were evaluated considering the classification quality reached using a rbf svm classifier. It was confirmed that a limited band subset was sufficient to classify common urban materials. The important contribution of bands from the Short Wave Infra-Red (SWIR) spectral domain (1000 - $2400 \mathrm{~nm})$ to material classification was also shown.
\end{abstract}

\section{INTRODUCTION}

\subsection{Some needs for urban materials maps}

During last decade, needs for high resolution land cover data have been growing. Indeed, such knowledge is necessary to answer several societal, regulatory and scientific needs, to produce environmental indicators to manage ecosystems and territories, to monitor environmental or human phenomena, or to be able to have a picture of an initial situation and to evaluate the impacts of public policies. Thus, to answer these needs, national mapping or environment agencies, in many countries, have undertaken the production of such large scale land cover databases. Nevertheless, these databases provide a general classification and may not suit some specific (often new) applications requiring a finer semantic or geometric level of details. That is to say that, on one hand, additional land cover classes should sometimes be specified, whereas, on the other hand, some existing classes should be delineated at a finer level.

Indeed, in urban areas, both semantic and spatial finer knowledge about land cover and especially maps of urban materials are required by several city modelling applications. Urban environment is indeed strongly influenced, in terms of ecology, energy and climate by the present materials. These materials can be either natural or artificial. Such material maps would be useful to derive indicators to monitor public policies impacts, or to feed urban simulation models. Indeed, at present, most applications

${ }^{*}$ Corresponding author are still experimental scientific ones such as micro-meteorology, hydrology, pollutants flow monitoring and ground perviousness monitoring. Several possible applications requiring very high resolution knowledge about urban land cover and materials are listed in (Heldens et al., 2011) and (Shafri et al., 2012) and described below.

1.1.1 Quantification of pollutant flows Some roofing materials can generate pollutant elements. Reducing the production of pollutants at their sources implies to identify sources and to quantify emissions. Several kinds of pollution are generated by roofing materials.

First, metallic elements are generated by corrosion of roof materials before being swept away by rainwater: roofing materials could be a major source of zinc, cadmium, lead and copper during wet weather (Chebbo et al., 2001). Especially, zinc emissions are mainly in the labile form (Heijerick et al., 2002), which is bioavailable and harmful to aquatic organisms. Copper roofs have also been identified as a possible source of pollution. Last, some other kinds of roofing materials can help to release organic polluting elements (polycyclic aromatic compounds, organic carbon) due to a not visible bitumen layer (Lemp and Weidner, 2005). Laboratory experiments have often already been done to model pollutant runoff rates for roofing materials (Robert-Sainte, 2009). Knowledge about the different roofing materials coverage areas is thus required so as to able to extrapolate these results to whole drainage areas: a map of roofing materials is thus needed. 
1.1.2 Monitoring of dangerous materials: asbestos-cement roofs Another possible application in the field of urban materials concerns the monitoring of asbestos-cement roofing materials (Heldens et al., 2011, Bassani et al., 2007). Asbestos-cement based materials can indeed be dangerous for human health, especially when they are deteriorated. Therefore, it is important to be at least able to evaluate the amount of buildings covered by asbestos-cement roofing sheets. Evaluating their deterioration status is also a useful issue.

1.1.3 Weather models Very fine knowledge concerning urban land cover (in terms of materials, perviousness and vegetation) are required by micro weather (wind, temperature, ...) model simulators (Heldens et al., 2011, Heldens et al., 2010, Kotthaus et al., 2014).

1.1.4 Monitoring of ground perviousness On one hand, it has been shown that the continuous development of impervious areas (especially in the periphery of cities), such as wide parking areas, plays an important role in the aggravation of flooding events, both in terms of magnitude and speed. Thus, having tools to monitor the extension of impervious areas and to check their appliance to new legislation would be useful. On the other hand, perviousness maps are required as input data by (micro) hydrological models (Heldens et al., 2011).

1.1.5 Determination of road type and monitoring of road condition At least, maps of road types (cobblestone, asphalt, ...) can be useful for some of the above mentioned applications. A more important and complex application focuses on the monitoring of road condition: such information indeed offers great interest for authorities in charge of the planning of road network renovation projects. Extracting this knowledge out of aerial data could be a way to avoid expensive and long field investigation (Herold et al., 2004b, Mohammadi, 2012).

1.1.6 Monitoring of photo-voltaic development On one hand, knowledge about roofing materials is a way to estimate the potential of a city to develop photo-voltaic energy (Roy, 2010, Jochem et al., 2009). On the other hand, detecting already installed panels is necessary to monitor the development of this technology.

\subsection{Toward a superspectral camera dedicated to urban ma- terial applications ?}

Thus, very high resolution urban land cover is required to provide knowledge about the roofing materials and the different kinds of ground areas. Such information can be a map of urban material (i.e. a classification). Since no existing map contains such information, airborne remote sensing techniques appear to be convenient for obtaining such a map at a large scale. However, remote sensing of urban environments from airborne acquisitions namely still remains a major issue, since on one hand, urban areas are characterised by a high variety of materials which can appear very similar on images, and on the other hand, by a strong intra-class variability due for instance to material aging and uses (Lacherade et al., 2005). Thus results provided by most traditional processing methods based on usual red-green-blue-near infrared multispectral images remain limited for such applications. A possible way to improve classification results is to enhance the imagery spectral resolution using superspectral or hyperspectral sensors.

Hyperspectral imagery consists of hundreds of contiguous spectral bands. Nevertheless, most of these spectral bands are highly correlated to each other and thus contain redundant information. Thus using all of them for a particular classification problem is not necessary. Therefore, only a subset of well selected spectral bands would be sufficient for urban materials classification
(Herold et al., 2004a). It would then be possible to design from this optimised band subset a superspectral aerial camera system dedicated to urban material classification. Such superspectral system could offer some advantages compared to most hyperspectral sensors. It could first make it possible to combine the use of suitable spectral bands for a specific application with a higher spatial resolution and a larger swath. It could also be a photogrammetric system, making it possible to capture multistereoscopic images, offering thus a possible calculation of BRDF models (Martinoty, 2005).

This paper presents experiments that were performed to define the optimal band subset for such superspectral sensor dedicated to urban material classification. The used automatic band selection framework and criterion are first presented. Second, data sets and experiments are described: experiments were performed on data sets generated from material reference reflectance spectra from available spectral libraries. These libraries and the way they were used to generate synthetic spectra are presented. Then, obtained results are presented, evaluated and discussed.

\section{SPECTRAL OPTIMISATION}

The selection of an optimal set of spectral bands is called spectral optimisation. To achieve this task, automatic feature selection (FS) methods can be used. FS methods will here be applied to select the most relevant band subset among the original bands of a hyperspectral data set for a specific classification problem.

\subsection{Feature selection: state-of-the-art}

Feature selection (FS) can be seen as a classic optimisation problem involving both a metric (that is to say a FS score measuring the relevance of feature subsets) to optimise and an optimisation strategy.

FS methods and criteria are often differentiated between "filter", "wrapper" and "embedded". It is also possible to distinguish supervised and unsupervised ones, whether classes are taken into account.

Filters Filter methods compute a score of relevance for each feature independently from any classifier. Some filter methods are ranking approaches: features are ranked according to a score of importance, as the ReliefF score (Kira and Rendell, 1992) or a score calculated from PCA decomposition (Chang et al., 1999). Other filters associate a score to feature subsets. In supervised cases, separability measures such as Bhattacharyya or JeffriesMatusita (JM) distances can be used in order to identify the feature subsets making it possible to best separate classes (Bruzzone and Serpico, 2000, Serpico and Moser, 2007). High order statistics from information theory such as divergence, entropy and mutual information can also be used to select the best feature subsets achieving the minimum redundancy and the maximum relevance, either in unsupervised or supervised situations: (Martínez-Usó et al., 2007) first cluster "correlated" features and then select the most representative feature of each group, while (Battiti, 1994, Estévez et al., 2009) select the set of bands that are the most correlated to the ground truth and the less correlated to each other.

Wrappers For wrappers, the relevance score associated to a feature subset corresponds to the classification performance (measured by a classification quality rate) reached using this feature subset. Examples of such approaches can be found in (Estévez et al., 2009, Li et al., 2011) using SVM classifier, (Zhang et al., 2007) using maximum likelihood classifier, (Díaz-Uriarte and De Andres, 2006) using Random Forests. 
Embedded Embedded FS methods are also related to a classifier, but feature selection is performed using a feature relevance score different from a classification performance rate. Some embedded approaches are regularisation models associating a fit-todata term (e.g. a classification error rate) to a regularisation function, penalising models when the number of features increases (Tuia et al., 2014). Other embedded approaches progressively eliminate features from the model, as SVM-RFE (Guyon et al., 2002) that considers the importance of the features in a SVM model. Other approaches have a built-in mechanism for feature selection, as decision trees using only the most discriminative feature when splitting a tree node (Breiman, 2001).

Another issue for band selection is the optimisation strategy to determine the best feature subset corresponding to a criteria. An exhaustive search is often impossible, especially for wrappers. Therefore, heuristics have been proposed to find a near optimal solution without visiting the entire solution space. These optimisation methods can be divided into incremental and stochastic ones.

Several incremental search strategies have been detailed in (Pudil et al., 1994), including the Sequential Forward Search (SFS) starting from one feature and incrementally adding another feature making it possible to obtain the best score or on the opposite the Sequential Backward Search (SBS) starting from all possible features and incrementally removing the worst features. Variants such as Sequential Forward Floating Search (SFFS) or Sequential Backward Search (SBFS) are proposed in (Pudil et al., 1994). Among stochastic optimisation strategies used for feature selection, several algorithms have been used for feature selection, including Genetic algorithms (Li et al., 2011, Estévez et al., 2009), Particle Swarm Optimisation (PSO) (Yang et al., 2012) or simulated annealing (De Backer et al., 2005, Chang et al., 2011).

\subsection{Proposed feature selection approach}

The proposed approach (Le Bris et al., 2014) relies on generic optimisation heuristics. It works in two steps (as reminded in fig. $1)$ :

1. First, the optimal number of spectral bands is identified using the incremental algorithm SFFS (Pudil et al., 1994). Indeed, in the context of sensor design, first step consists in optimising the number of band. SFFS starts from empty band subset, and incrementally add bands to the subset, considering a FS score, and questioning the current band subset solution each time a new band is selected. Thus, this algorithm makes it possible to see the influence of the number of selected bands on the classification results.

2. Optimised band subsets solutions are then proposed genetic algorithm (GA) for the optimal number of bands identified at previous step. GA is a stochastic algorithm and it is here used to provide several good solutions. At the end, the solution involving the less correlated bands to each other is retained as the final solution. Besides, intermediate good band subsets candidates proposed by GA are used to derive band importance profiles, assessing the importance of bands considering the frequency at which they appeared among these intermediate solutions.

\subsection{Used FS criterion}

The score used to evaluate the relevance of band subsets within the previous framework is adapted from the one proposed in (Le Bris et al., 2015). It is a wrapper score that relies on Random Forests classifier and takes into account classification confidence.
Hyperspectral data + ground truth
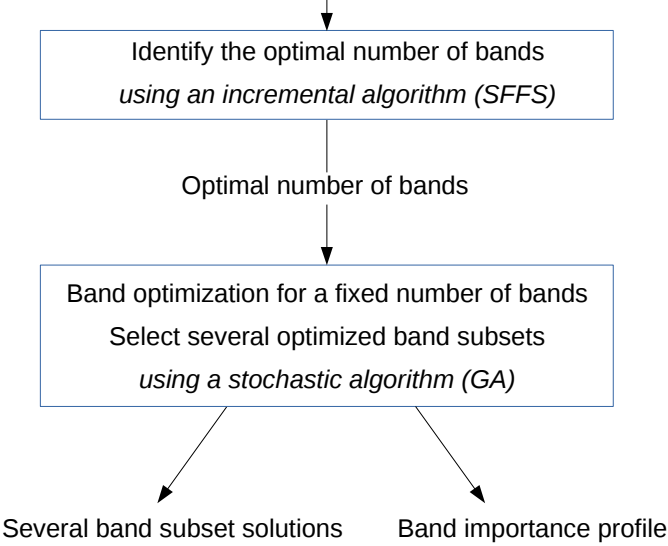

Band importance profile

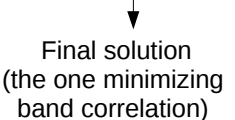

Figure 1: Proposed feature selection approach

2.3.1 Random Forests Random Forests (RF) (Breiman, 2001) is a modification of bagging applied with decision trees. It can achieve a classification accuracy comparable to boosting (Breiman, 2001), or SVM (Pal, 2005). It does not require assumptions on the distribution of the data, which is interesting when different types or scales of input features are used. It was successfully applied to remote sensing problems involving multispectral, hyperspectral or multisource data. This ensemble classifier is a combination of tree predictors built from $T$ multiple bootstrapped training samples. For each node of a tree, a subset of features is randomly selected. Then, the best feature with regard to Gini impurity measure is used for node splitting. For classification, each tree gives a vote for the most popular class at each input instance and the final label is determined by a majority vote of all trees. Thus, for each sample to classify, the number of votes obtained by each possible label can be used as a class membership measure. Besides, it is provided by Random Forests at no additional computational cost.

Let $\mathcal{C}=\left\{c_{1}, \ldots, c_{n c}\right\}$ be the set of possible classes and $v(\mathbf{x}, c)$ the number of votes obtained by class $c$ when classifying sample $\mathbf{x}$. A class membership score $m$ can then be obtained by normalising the number of votes by the number of trees: $m(\mathbf{x}, c)=\frac{v(\mathbf{x}, c)}{T}$. $\mathrm{RF}$ also provides a classification confidence measure named unsupervised margin and defined as the difference between the two best class memberships, that is to say:

$$
\mathcal{M}(\mathbf{x})=m(\mathbf{x}, \hat{c})-\max _{c \neq \hat{c}} m(\mathbf{x}, c) \quad \text { with } \quad \hat{c}=\operatorname{argmax}_{c} m(\mathbf{x}, c)
$$

The more confident the classifier, the more the margin.

2.3.2 A FS score taking into account RF confidence measures Let $\mathcal{X}=\left\{\left(\mathbf{x}_{i}, y_{i}\right)\right\}_{1 \leq i \leq n}$ be a set a ground truth samples $\mathbf{x}_{i}$ and their associated true label $y_{i}$. A possible feature selection score $\mathcal{R}$ taking into account class membership measures and thus classification confidence can be defined as:

$$
\mathcal{R}(\mathcal{X})=\sum_{i=1}^{n} \delta\left(y_{i}, c\left(\mathbf{x}_{i}\right)\right) \cdot \mathcal{M}\left(\mathbf{x}_{i}\right) \in[0 ; 1]
$$

with $\delta(i, j)=\{-1$ if $i \neq j$ and 1 otherwise $\}$, and $c(\mathbf{x})$ the label given to $\mathbf{x}$ by the classifier. 
This score has the advantage to measure both the ability to well classify the test samples for a given feature set and the separability between classes. Indeed, the more the samples are well classified, the more the score increases. The more the classifier is confident for well classified samples, the more the score increases. The more the classifier is confident for bad labelled samples, the more the score decreases.

\section{DATA SET}

Spectral optimisation was performed from a library of reference spectra of urban materials. These spectra were collected from several available existing spectral libraries listed in section 3.1. Such data offers several advantages, compared to another alternative such as the use of aerial hyperspectral scenes over several urban landscapes. On one hand, these spectra were captured through field or laboratory measurements, and are thus pure and "clean" reflectance measures. On the other hand, they are generally well described, without ambiguity about their class. Besides, it is a way to have spectra of rare (but thematically important) materials.

\subsection{Spectral libraries}

The reference spectra used in this study were collected from several available existing spectral libraries. The number of spectra per original library is shown by table 1 .

- ASTER Spectral Library ${ }^{1}$ : The ASTER spectral library (Baldridge et al., 2009) is made available by the Jet Propulsion Laboratory. It contains more than 2400 spectra of natural and artificial materials from 3 other spectral libraries: the Johns Hopkins University (JHU) Spectral Library, the Jet Propulsion Laboratory (JPL) Spectral Library and the USGS Spectral Library.

- SLUM ${ }^{2}$ : The Spectral Library of impervious Urban Materials (SLUM) (Kotthaus et al., 2014) is produced within the London Urban Micromet data Archive (LUMA). It contains reflectance spectral measures of 74 impervious materials collected in London.

- MEMOIRES ${ }^{3}$ and ONERA data : Many urban materials spectra were made available by ONERA, and especially from the spectral library MEMOIRES (Moyen d'Echange et de valorisation de Mesures de propriétés thermiques, Optiques et InfraRouges d'Echantillons et de Scènes) (Martin and Rosier, 2012). Most of them were collected in Toulouse (France).

- Santa Barbara libraries ${ }^{4}$ : Many urban materials spectra collected (only field measures) on Santa Barbara (Herold et al., 2004a) are available. Two libraries can be distinguished: one dedicated to spectral optimisation for urban classification (Herold et al., 2004a) and the other dedicated to the analysis of road conditions (Herold et al., 2004b).

- Ben Dor spectral libray Spectra collected in Tel Aviv by (Ben-Dor et al., 2001) for urban classification were also used.

- DESIREX Spectra from field measurements campaign DESIREX 08 (ESA) (Sobrino, 2008) in Madrid were also available.

\footnotetext{
${ }^{1}$ http://speclib.jpl.nasa.gov/

${ }^{2} \mathrm{http} / / /$ LondonClimate.info/LUMA/SLUM.html

${ }^{3} \mathrm{http}: / / \mathrm{www}$. onera.fr/dota/memoires

${ }^{4}$ http://www.ncgia.ucsb.edu/ncrst/research/pavementhealth/urban/
}

Table 1: Origin of collected spectra

\begin{tabular}{|l|r|}
\hline Library & Number of spectra \\
\hline ASTER & 29 \\
BenDor & 33 \\
SLUM & 55 \\
DESIREX & 11 \\
ONERA MEMOIRES & 282 \\
SantaBarbara_road & 43 \\
SantaBarbara_materials & 62 \\
\hline
\end{tabular}

\subsection{Integration of collected spectra into a data base}

First, all collected spectra were integrated into a common data base. This required to define a common legend, to be able to have a homogeneous spectral collection. Interesting taxonomies for urban materials have been proposed in previous works such as (Heiden et al., 2007) or (Herold et al., 2004a). These taxonomies are often hierarchical ones, with a last level of detail corresponding to fine information about materials such as colour or condition. However, at this step, it is intended to keep as many information as possible to describe the collected spectra rather than to have a frozen nomenclature. Thus, it was decided to store collected spectra in our database, associating several attributes to each of them:

- Material class

- Variety (e.g. "zinc" or "steel" for material "metal")

- Colour

- Condition (e.g. aging)

- Corresponding land cover (e.g. "ground" or "roof" for "gravels")

It must here be kept in mind that it was not always possible to obtain all these information for most spectra.

Spectral domain: Only spectra concerning both the Visible Near Infra-Red (VNIR) (400-1000 nm) and the Short Wave Infra-Red (SWIR) (1000-2400 nm) spectral domains were kept. The spectral resolution of the collected spectra was generally comprised between 1 and $5 \mathrm{~nm}$, and sometimes $10 \mathrm{~nm}$ in the SWIR domain. Besides, all collected spectra had not been measured under the same conditions. Only reflectance spectra were considered. However, for spectral optimisation experiments, it was necessary to remove the bands concerned by atmospheric absorption. Furthermore, other artefacts were present on some spectra, as for instance some transitions between the VNIR and SWIR sensor of an ASD. They also had to be removed.

Ignored classes: Experiments focused on artificial materials. Thus, some classes were let aside from the data base, even if they can be important in urban land cover. For instance, vegetation is a key element in urban landscape, but was let aside in next experiments, since the discrimination between vegetation and non vegetation is easy and because it was intended to be considered in further studies specifically dedicated to its characterisation. Water was also let aside, since few spectra were available and because its aspect can be very different depending on depth, turbidity and eutrophysation level. On the opposite, natural bare ground was considered since it is very important in perviousness studies.

At the end, the synthesis of the kept spectra is presented on figure 2. It can be seen that there is a strong heterogeneity in the number of available spectra per class: some classes (asphalt, concrete, stone pavements) are well represented, while other ones concern very few spectra (such as slates and asbestos). Furthermore, the number of spectra per class is generally not sufficient to correctly 
evaluate intra class variability and thus to be significant to perform spectral optimisation using the proposed method, and to validate results on test data sets.

To cope with this insufficient number of available spectra, it was here proposed to generate new spectra from the ones in the data base.

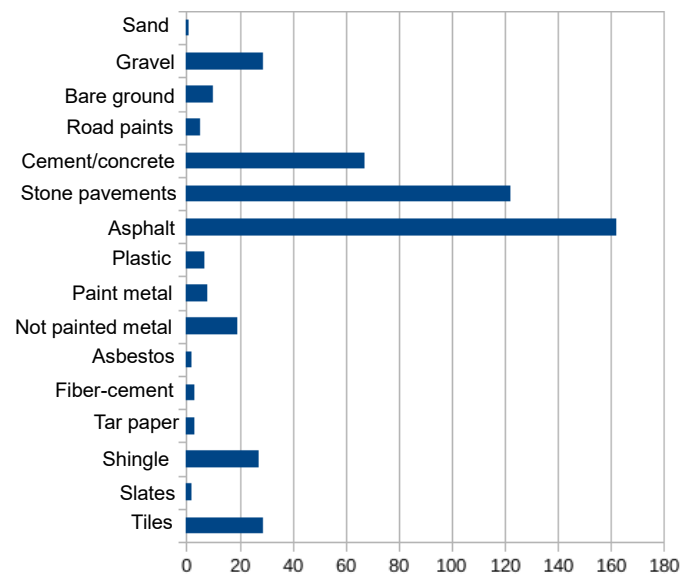

Figure 2: Number of available spectra for the most important material classes

\subsection{Generate new synthetic spectra from the data base}

A random multiplicative factor was simply applied to reference spectra in order to generate more synthetic spectra from the data base (DB). It partly simulates intra-class variability, even though it does not simulate the totality of intra-class variability (such as colour or aging). For each generated synthetic spectrum, the multiplicative factor was randomly selected between 0.8 and 1.2, according to the standard deviations of the classes for which a sufficient amount of spectra was available. Finer quantitative analyses are available in (Lacherade et al., 2005).

At the end, the proposed process to generate an experimental data set (also reminded in fig. 3 ) is as follows:

For each class c do

Generate a set of synthetic spectra $G T_{c}$ for class $c$ :

$G T_{c} \leftarrow \emptyset$

Create a query to list the spectra belonging to this class

Create list $L_{c}$ of spectra from the DB corresponding to this query

For i from 1 to $n$ do

Randomly select spectrum s from $L_{c}$

Variability generation:

Apply to s a random multiplicative factor (between 0.8 and 1.2) $s \leftarrow \operatorname{rand}() . s$

Add this spectrum to the experimental data set: $G T_{c} \leftarrow G T_{c} \cup s$ EndFor

EndFor

\section{EXPERIMENTS AND RESULTS}

Experiments were performed for next legend. It consisted in classes corresponding both to the most common materials in the database and to other important classes (e.g. slate) frequently present in urban areas. Such classes would be the basic classes of a material map, because they are likely sure to be found in an urban area.

- Slate

- Asphalt

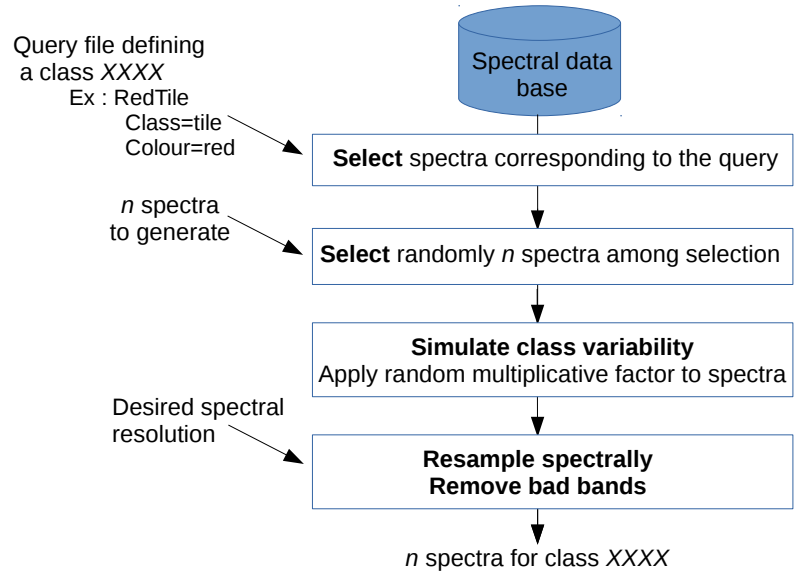

Figure 3: Synthetic spectra collection generation scheme

- Cement/concrete

- Gravel

- Metal

- Stone pavement bricks

- Shingle

- Bare ground

- Tile

In order to perform spectral optimisation, a data set was generated from the data base according to this legend. It contained 100 training spectra and 500 test spectra, resampled at a $10 \mathrm{~nm}$ spectral resolution ranging from 420 to $2400 \mathrm{~nm}$.

\subsection{Band selection in the [VNIR-SWIR] domain $(420-2400$} nm)

Band selection was first performed within the spectral domain ranging from 420 to $2400 \mathrm{~nm}$.

First, the optimal number of spectra was defined, owing to SFFS algorithm. Figure 4 shows the evolution of the FS score and of several classification quality rates reached by a RBF SVM classifier depending on the number of selected bands. It can be seen that up to a band subset size, selecting new bands has very few impact on results. Thus, in next experiments 10 bands were selected.

Then, several 10-band subsets (presented in figure 5 ) were proposed by GA. Band importance profile (displayed on figure 5) was calculated from intermediate results of GA. Some part of the spectrum were considered relevant, especially in the VNIR domain and in the 2000-2400 nm range of the SWIR domain, while, on the opposite, the $1000-1500 \mathrm{~nm}$ spectral domain is not considered relevant for this classification task.

At the end, the band subset with the less correlated bands was selected and evaluated.

\subsection{Band selection in the VNIR domain $(420-1000 \mathrm{~nm})$}

The same process was applied to the $420-1000 \mathrm{~nm}$ spectral domain. An optimal number of 10 bands was also identified by SFFS. Then, several band subsets (presented in figure 6 ) were proposed by GA for 10 bands, and band importance profile (displayed on figure 6) was calculated from intermediate results of GA. Although 10 bands were selected, a pattern of 4-5 important blobs appears along the spectrum, corresponding approximately to usual multispectral bands (blue, green, red and near infrared). 

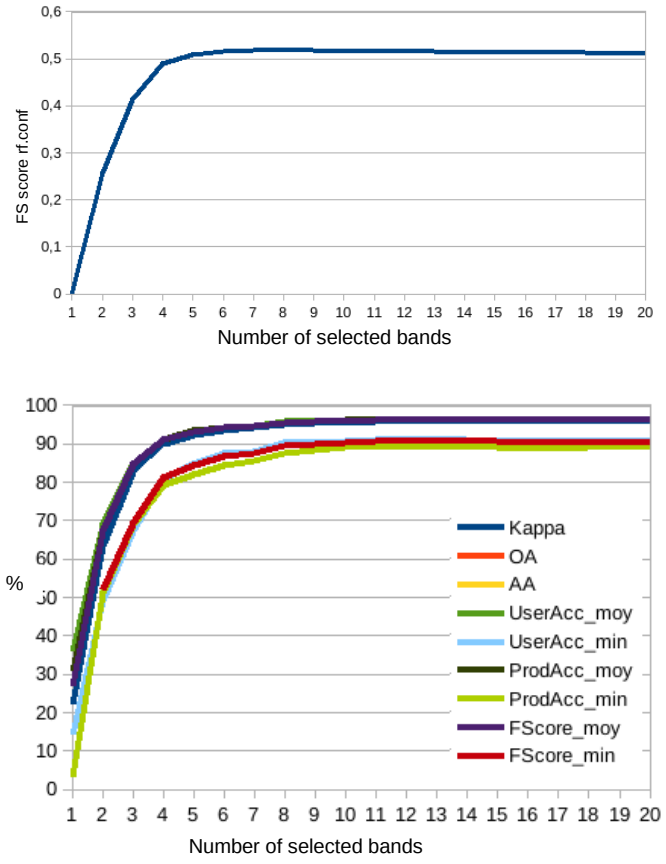

Figure 4: Evolution of the FS score (top) and of the quality of RBF SVM classification depending on the number of selected bands
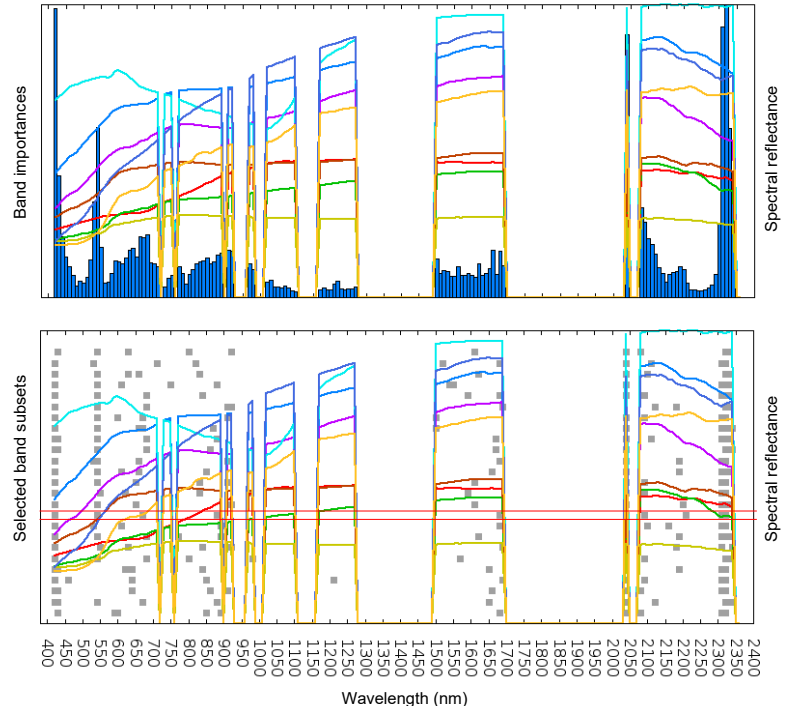

Figure 5: Selected band subsets of 10 bands (top) and band importance profiles (bottom) for spectral optimisation in the [VNIRSWIR] $(420-2400 \mathrm{~nm})$ spectral domain. (red frame = final solution)

\subsection{Evaluation of optimised subsets of bands from different spectral domains}

The classification performance of the band subsets previously optimised was evaluated for a RBF SVM classifier. The classifier was applied to a test data set containing 1000 samples per class. Two scenarios were considered to train the classifier using either 100 or 50 samples per class, so as to have an easy case and a more difficult one.

This quantitative evaluation was first performed both for the whole
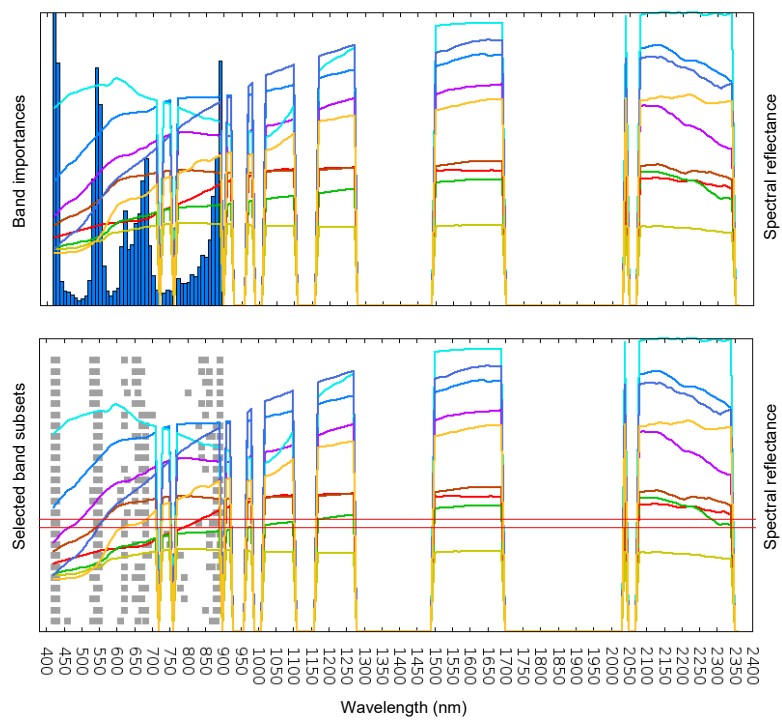

Figure 6: Selected band subsets of 10 bands (top) and band importance profiles (bottom) for spectral optimisation in the VNIR $(420-1000 \mathrm{~nm})$ spectral domain. $($ red frame $=$ final solution $)$

hyperspectral set of bands and for the subsets of 10 bands selected from the VNIR (420-1000 nm) and from the [VNIR-SWIR] (420$2400 \mathrm{~nm}$ ) spectral domains, but also from the [VNIR-SWIR] spectral domain limited to the $420-1800 \mathrm{~nm}$ range. Indeed, it was interesting to evaluate the impact of a restriction to the first part of the SWIR domain, since it is less perturbed by atmospheric effects and receives more photons than the (1800-2400 nm) part. Results are presented on figure 7: 10 bands selected in the 420$2400 \mathrm{~nm}$ domain led to similar results as when using all the hyperspectral bands. The worst results were obtained for band subsets limited to the VNIR domain, while intermediate results were reached using bands from the $420-1800 \mathrm{~nm}$. The differences between the classification precisions reached for the different spectral configurations tended to be more significant for the difficult training scenario (i.e. when the classifier is trained from only 50 samples per class), since Kappa is 0.90 for all bands, 0.90 for 10 bands from the [VNIR-SWIR] (420-2400 nm), 0.87 for 10 bands from the $(420-1800 \mathrm{~nm})$ range and 0.81 for 10 bands from the VNIR domain.

Further experiments were performed to assess the relevance of bands from the SWIR domain for urban materials classification: only 4 individual original bands were selected (as for usual multispectral sensors). As for previous experiments, subsets of 4 bands were selected from the VNIR (420-1000 nm), the [VNIRSWIR1] (420-1800 nm) and the the [VNIR-SWIR] (420-2400 $\mathrm{nm})$ spectral domains. Their classification performances for a RBF SVM classifier were compared to the configuration of an existing multispectral sensor: the Pléiades satellite. Results are presented on figure 8 . As previously, best results were obtained using bands selected in the [VNIR-SWIR] domain. Indeed, when the classifier is trained from 50 samples per class, Kappa reached 0.82 for 4 bands from the [VNIR-SWIR] $(420-2400 \mathrm{~nm}), 0.81$ for 4 bands from the $(420-1800 \mathrm{~nm})$ range and 0.78 for 4 bands from the VNIR domain. It can also be said that better results were reached using the optimised subset of 4 bands from the VNIR domain rather than the Pléiades configuration, for which Kappa reached 0.74 .

Nevertheless, obtained quantitative evaluations are really optimistic and must be considered carefully. Indeed, it must be kept in mind that some classes were represented by very few spectra 
in the spectral data base, and thus, their variability is not completely considered. For instance, "slates" represented by few spectra in the data base were very well classified, while on the opposite some classes, such as "asphalt", "cement/concrete" or "stone pavements", which were represented by larger amounts of reference spectra in the data base obtained the lowest classification rates. Thus, there is also a risk of overfitting.

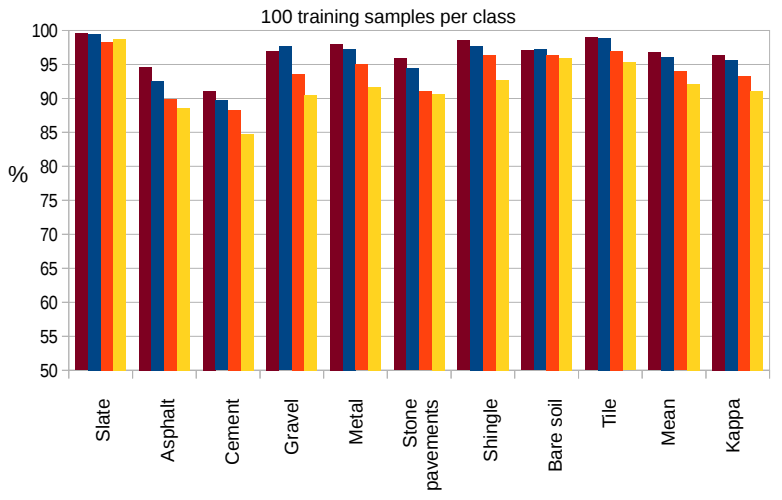

- Hyperspectral 10 bands from [VNIR-SWIR] (420-1800 nm) - 10 bands from [VNIR-SWIR] (420-2400 nm) 10 bands from VNIR $(420-1000 \mathrm{~nm})$

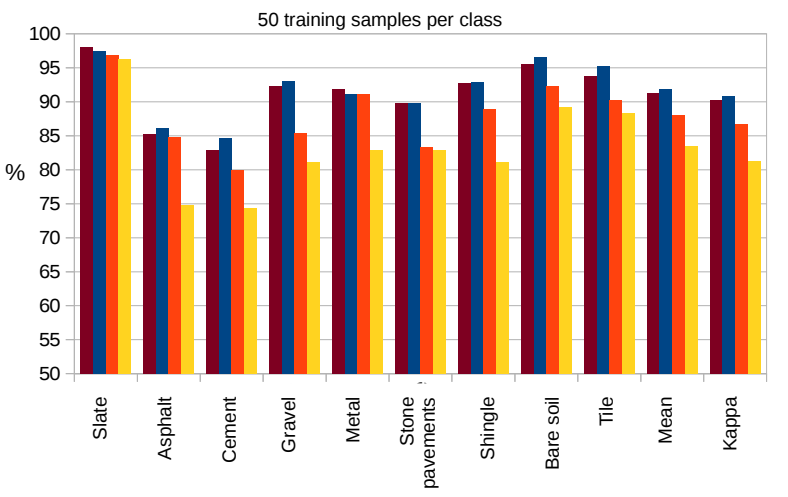

Figure 7: Quantitative results reached using selected band subsets of 10 bands from different spectral domains: F-scores of the different classes and Kappa coefficient reached by RBF SVM classification. The classifier was trained using 100 samples (top) and 50 samples (bottom).

\section{CONCLUSION}

In this study, band selection was performed to identify optimal band subsets for urban map classification in the context of designing a superspectral sensor dedicated to this application.

Spectral optimisation was performed on data sets generated from a collection of reference reflectance spectra from several available spectral libraries.

A limited number of bands (10) was proven to be sufficient to obtain good discrimination between 9 common urban materials. The importance of the SWIR domain (and especially of the 1800$2400 \mathrm{~nm}$ ) was also confirmed.

Nevertheless, some classes were represented by very few spectra in the spectral data base, and thus, their variability can not be completely considered. Therefore, obtained quantitative evaluations are really optimistic and must be considered carefully. However, new urban material spectra measurement campaigns will occur within the French ANR HYEP ${ }^{5}$ project and will be integrated in the data base. Besides, further experiments will also be carried out using aerial hyperspectral scenes. They will bring

\footnotetext{
${ }^{5}$ http://hyep.cnrs.fr
}
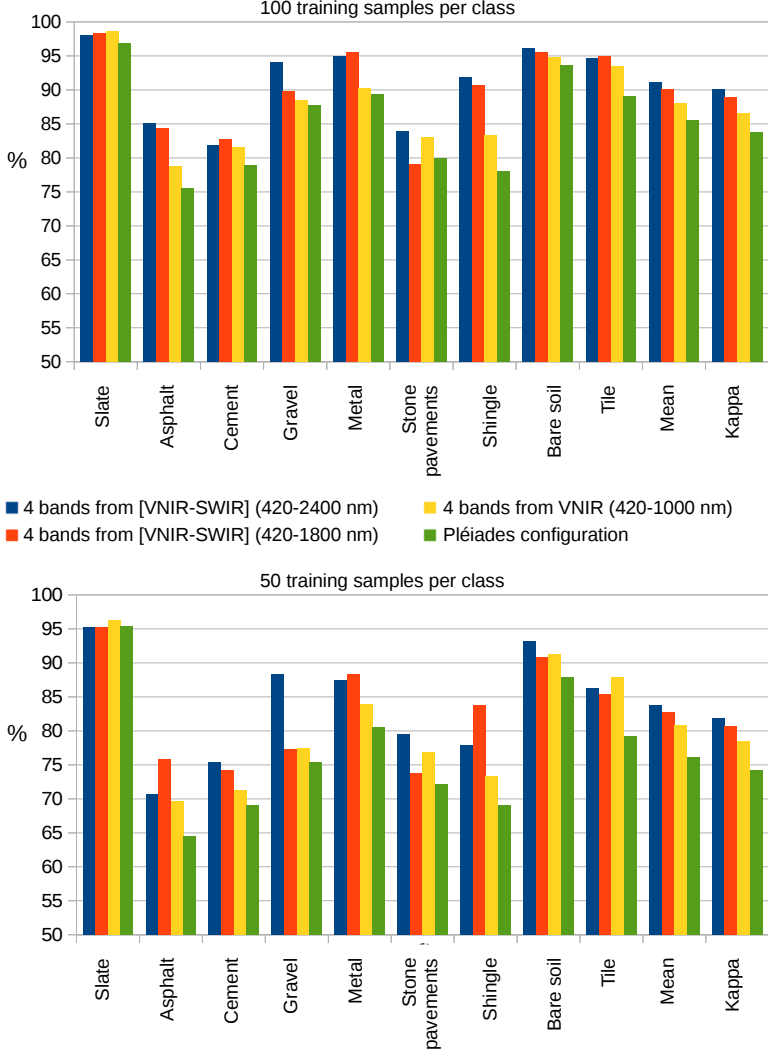

Figure 8: Quantitative evaluation of results reached using selected band subsets of 4 bands from different spectral domains: F-scores of the different classes and Kappa coefficient reached by RBF SVM classification. The classifier was trained using 100 samples (top) and 50 samples (bottom).

more realistic evaluation results, since such data will be perturbed by sensor and atmospheric noise, while in the experiments presented in this paper such phenomena were not taken into account since only clean reflectance data measured on the field or in laboratory were used.

\section{REFERENCES}

Baldridge, A. M., Hook, S., Grove, C. and Rivera, G., 2009. The ASTER spectral library Version 2.0. Remote Sensing of Environment 113, pp. 711-715.

Bassani, C., Cavalli, R., Cavalcante, F., Cuomo, V., Palombo, A., Pascucci, S. and Pignatti, S., 2007. Deterioration status of asbestos-cement roofing sheets assessed by analyzing hyperspectral data. Remote Sensing of Environment 109, pp. 361-378.

Battiti, R., 1994. Using mutual information for selecting features in supervised neural net learning. IEEE Transactions on Neural Networks.

Ben-Dor, E., Levin, N. and Saaroni, H., 2001. A spectral based recognition of the urban environment using the visible and nearinfrared spectral region $(0.4-1.1 \mu \mathrm{m})$. A case study over TelAviv, Israel. International Journal of Remote Sensing 22(11), pp. 2193-2218.

Breiman, L., 2001. Random forests. Machine Learning 45(1), pp. 5-32.

Bruzzone, L. and Serpico, S. B., 2000. A technique for feature selection in multiclass problem. International Journal of Remote Sensing 21(3), pp. 549-563. 
Chang, C.-I., Du, Q., Sun, T.-L. and Althouse, M., 1999. A joint band prioritization and band-decorrelation approach to band selection for hyperspectral image classification. IEEE Transactions on Geoscience and Remote Sensing 37(6), pp. 2631-2641.

Chang, Y.-L., Chen, K.-S., Huang, B., Chang, W.-Y., Benediktsson, J. and Chang, L., 2011. A parallel simulated annealing approach to band selection for high-dimensional remote sensing images. IEEE Journal of Selected Topics in Applied Earth Observations and Remote Sensing 4(3), pp. 579-590.

Chebbo, G., Gromaire, M., Ahyerre, M. and Garnaud, S., 2001. Production and transport of urban wet weather pollution in combined sewer systems: the Marais experimental catchment in paris. Urban Water 3(1-2), pp. 3-15.

De Backer, S., Kempeneers, P., Debruyn, W. and Scheunders, P., 2005. A band selection technique for spectral classification. IEEE Geoscience and Remote Sensing Letters 2(3), pp. 319-232.

Díaz-Uriarte, R. and De Andres, S. A., 2006. Gene selection and classification of microarray data using random forest. BMC bioinformatics 7(3), pp. 1-13.

Estévez, P. A., Tesmer, M., Perez, C. A. and Zurada, J. M., 2009. Normalized mutual information feature selection. IEEE Transactions on Neural Networks 20(2), pp. 189-201.

Guyon, I., Weston, J., Barnhill, S. and Vapnik, V., 2002. Gene selection for cancer classification using support vector machines. Machine Learning 46, pp. 289-422.

Heiden, U., Segl, K., Roessner, S. and Kaufmann, H., 2007. Determination of robust spectral features for identification of urban surface materials in hyperspectral remote sensing data. Remote Sensing of Environment 111(4), pp. $537-552$.

Heijerick, D., Janssen, J., Karlen, C., Odnevall Wallinder, I. and Leygraf, C., 2002. Bioavailability of zinc in runoff water from roofing materials. Chemosphere 47, pp. 1073-1080.

Heldens, W., Heiden, U., Esch, T. and Dech, S., 2010. Potential of hyperspectral data for urban micro climate analysis. In: Proc. of the Hyperspectral 2010 Workshop.

Heldens, W., Heiden, U., Esch, T., Stein, E. and Muller, A., 2011. Can the future EnMAP mission contribute to urban applications? a literature survey. Remote Sensing 3, pp. 1817-1846.

Herold, M., Roberts, D., Gardner, M. and Dennison, P., 2004a. Spectrometry for urban area remote sensing-development and analysis of a spectral library from 350 to $2400 \mathrm{~nm}$. Remote Sensing of Environment 91(3-4), pp. $304-319$.

Herold, M., Roberts, D., Smadi, O. and Noronha, V., 2004b. Road condition mapping using hyperspectral remote sensing. In: Proceedings of the AVIRIS Workshop, Pasadena, USA.

Jochem, A., Höfle, B., Rutzinger, M. and Pfeifer, N., 2009. Automatic roof plane detection and analysis in airborne lidar point clouds for solar potential assessment. Sensors 9, pp. 5241-5262.

Kira, K. and Rendell, L., 1992. A practical approach to feature selection. In: Proceedings of the 9th International Workshop on Machine Learning, pp. 249-256.

Kotthaus, S., Smith, T., Wooster, M. J. and Grimmond, C., 2014. Derivation of an urban materials spectral library through emittance and reflectance spectroscopy. ISPRS Journal of Photogrammetry and Remote Sensing 94, pp. 194-212.

Lacherade, S., Miesch, C., Briottet, X. and Le Men, H., 2005. Spectral variability and bidirectional reflectance behaviour of urban materials at a $20 \mathrm{~cm}$ spatial resolution in the visible and nearinfrared wavelengths. a case study over Toulouse (France). International Journal of Remote Sensing 26(17), pp. 3859-3866.

Le Bris, A., Chehata, N., Briottet, X. and Paparoditis, N., 2014. Identify important spectrum bands for classification using importances of wrapper selection applied to hyperspectral data. In: Proc. of the 2014 International Workshop on Computational Intelligence for Multimedia Understanding (IWCIM'14).
Le Bris, A., Chehata, N., Briottet, X. and Paparoditis, N., 2015. A Random Forest class memberships based wrapper band selection criterion : application to hyperspectral. In: Proc. of the IEEE International Geoscience and Remote Sensing Symposium (IGARSS).

Lemp, D. and Weidner, U., 2005. Improvements of roof surface classification using hyperspectral and laser scanning data areas. International Archives of Photogrammetry, Remote Sensing and Spatial Information Sciences 36(8/W27), pp. CD-Rom.

Li, S., Wu, H., Wan, D. and Zhu, J., 2011. An effective feature selection method for hyperspectral image classification based on genetic algorithm and support vector machine. Knowledge-based Systems 24, pp. 40-48

Martin, C. and Rosier, B., 2012. Status of "MEMOIRES" - a thermal and optical data base (Mean of Exchange and valorization of Measurements of thermal, Optical and InfraREd properties of Samples and scenes. In: Fraunhofer IOSB, Ettlingen, Allemagne.

Martínez-Usó, A., Pla, F., Martínez Sotoca, J. and García-Sevilla, P., 2007. Clustering-based hyperspectral band selection using information measures. IEEE Transactions on Geoscience and Remote Sensing 45(12), pp. 4158-4171.

Martinoty, G., 2005. Reconnaissance de matériaux sur des images aériennes en multirecouvrement, par identification de fonctions de réflectance bidirectionnelles. $\mathrm{PhD}$ thesis, Université Paris 7 - Denis Diderot.

Mohammadi, M., 2012. Road classification and condition determination using hyperspectral imagery. International Archives of the Photogrammetry, Remote Sensing and Spatial Information Sciences.

Pal, M., 2005. Random forest classifier for remote sensing classification. International Journal of Remote Sensing 26(1), pp. 217 222.

Pudil, P., Novovicova, J. and Kittler, J., 1994. Floating search methods in feature selection. Pattern Recognition Letters 15, pp. 1119-1125.

Robert-Sainte, P., 2009. Contribution des matériaux de couverture à la contamination métallique des eaux de ruissellement. $\mathrm{PhD}$ thesis, Université Paris-Est.

Roy, J., 2010. The use of VHR remote sensing imagery for the identification of roofs potentially suitable for the installation of photovoltaic panels. Présentation aux Journées Thématiques Pléiades.

Serpico, S. B. and Moser, G., 2007. Extraction of spectral channels from hyperspectral images for classification purposes. IEEE Transactions on Geoscience and Remote Sensing 45(2), pp. 484495 .

Shafri, H., Taherzadeh, E., Mansor, S. and Ashurov, R., 2012. Hyperspectral remote sensing of urban areas: an overview of techniques and applications. Research Journal of Applied Sciences, Engineering and Technology 4(11), pp. 1557-1565

Sobrino, J. e., 2008. Desirex 2008 final report - dual-use european security ir experiment 2008 (desirex 2008). Technical report, ESA.

Tuia, D., Volpi, M., Dalla Mura, M., Rakotomamonjy, A. and Flamary, R., 2014. Automatic feature learning for spatio-spectral image classification with sparse svm. IEEE Transactions on Geoscience and Remote Sensing 52(10), pp. 6062-6074.

Yang, H., Du, Q. and Chen, G., 2012. Particle swarm optimization-based hyperspectral dimensionality reduction for urban land cover classification. IEEE Journal of Selected Topics in Applied Earth Observations and Remote Sensing 5(2), pp. 544-554.

Zhang, L., Zhong, Y., Huang, B., Gong, J. and Li, P., 2007. Dimensionality reduction based on clonal selection for hyperspectral imagery. IEEE Transactions on Geoscience and Remote Sensing 45(12), pp. 4172-4186. 